\title{
REPORTE DEL PRIMER CASO DE RINOSPORIDIOSIS EN LA PROVINCIA CIEGO DE AVILA (CUBA)
}

\author{
(First case report of rhinosporidiosis in the Ciego de Avila Province - Cuba)
}

\author{
M. Suarez, $H^{*}$, J. Rodriguez, F.**, \\ A. Duarte, $\mathbf{V}^{* *}$, V.M.Lopez, $\mathbf{P}^{* *}, \mathbf{A}$. Bermudez, $\mathbf{B}^{* *}$, \\ E. Salcedo, $\mathbf{R}^{* *}, \mathbf{R}$. Pelaez, $\mathbf{M}^{* * *}$. \\ *Centro Provincial de Higiene y Epidemiología, Ciego de Avila. \\ ** Hospital Provincial Docente""'Antonio Luases Iraola" Ciego de Avila \\ *** Facultad de Ciencias Médicas de Ciego de Avila.. CUBA.
}

Palabras clave: Micosis, Rinosporidiosis

Key Words: Mycoses, Rhinosporidiosis

\section{RESUMEN}

Se presenta el primer caso de rinosporidiosis diagnosticado en la provincia Ciego de Avila,Cuba, analizandose las caracteristicas clinico-epidemiológicas del caso. Paciente de 13 años de edad, de sexo masculino, proveniente de un área rural que presentaba localización nasal de las lesiones. Se revisa la literatura al respecto.

\section{INTRODUCCION}

La Rinosporidiosis es una enfermedad micótica producida por el Rinosporidium seeberi. Este microorganismo no se ha podido cultivar para su estudio. La entidad es éndemica de la India pero es rara en Europa y otros continentes (1).

En 1971 en Cuba, se reportó un caso en la conjuntiva ocular en un paciente de 56 años (9).

No se conoce la fuente de infección en la naturaleza, se sospecha que este agente penetra en el hombre a través de partículas del suelo por las mucosas lesionadas. En los lugares en que es endémica, esta enfermedad se ha asociado a las aguas estancadas, pero hasta el presente no se ha podido demostrar la presencia de este agente biológico en la misma (2).

$\mathrm{Su}$ ciclo vital es reducido y se basa fundamentalmente en estudios sobre cortes histológicos de tejidos. SE presentan esporangios en los tejidos de la mucosa nasal de aproximadamente unos 200-300 $\mu \mathrm{m}$ de diámetro, con gruesa pared que consta de 2 capas: una externa quitinosa y una interna que contiene celulosa.

\section{SUMMARY}

The first case report of rhinosporidiosis diagnosed in the province of Ciego de Avila, Cuba, is submitted here together with an analysis of the clinical-epidemiological characteristics. It is a 13 year-old male patient, coming from a country area whose lesions were located on his nose. Suitable literature is also reviewed.

Con el tiempo, mediante clivaje de su citoplasma y sucesisvas mitosis nucleares, se forman endosporas de 7$9 \mu \mathrm{m}$ de diámetro que se liberan por ruptura de la pared. Las esporas liberadas están rodeadas por material granular mucoide y permanecen en el epitelio que a su vez forman nuevos esporangios para completar el ciclo de la enfermedad.

Los esporangios son visibles a simple vista como unas manchas blancas en las lesiones. La Rinosporidiosis, se presenta frecuentemente como un pólipo o una masa polipoide algo friable en la que pueden observarse pequeñas manchas en la superficie, lo que generalmente permite su diferenciación de algunos pólipos alérgicoso inflamatorios.

La nariz es el sitio más frecuente de infección, aunque la laringe, faringe, oídos, piel, genitales externos, recto y otras mucosas pueden estar comprendidas $(3,4,5)$. El diagnóstico depende de la apariencia clínica y de la demostración del agente biológico en los tejidos por examen directo del material de las masas polipoides (utilizando una gota de agua o de solución salina), en las cuales se revela la presencia de esporangios. Otra forma de diagnóstico es la biopsia, teñidas con los métodos de PAS o GMS, en la cual pueden observarse en los cortes histológicos, esporangios, llenos de numerosas endosporas 
$(5,6,7)$.

Nuestros objetivos fueron: la presentación de un caso clínico, su evolución y estudio epidemiológico.

\section{PRESENTACION DEL CASO}

A.M.Q., de 13 años de edad, raza blanca, residente en el área norte de la provincia de Ciego de Avila ,de profesión estudiante. El paciente acude a la consulta de otorrinolaringología por presentar hace 2 meses epistaxis unilateral derecha, no abundante, sin antecedentes de trauma ni infección previa en la naríz. La mucosa nasal estaba congestionada y se observaba una formación de aspecto redondeado polipoide en el vestibulo nasal inferior derecho. La tumoración midió aproximadamente 1 $\mathrm{cm}$., de color rosado, sangreante facilmente al tacto, no siendo dolorosa. Se indicó cirugía menor y luego una muestra se envió para estudiohistopatológico. El paciente evolucionó satisfactoriamente con buena cicatrización aparente de las lesiones.

En el contról postoperatorio (21 días), se observó recidiva, indicandose nuevamente extirpación y cauterización con nitrato de plata. El resultado de la biopsia demostró la presencia de Rhinosporidium seeberi.

En el estudio epidemiológico realizado, se detectó que el paciente no tuvo antecedentes de trauma nasal, además en la finca donde reside existían caballos y perros, los cuales no tenían síntomas Los informes del servicio veterinario aseveraron la ausencia de animales con lesiones por este microorganismo.

\section{RESULTADO Y DISCUSION}

El caso analizado corresponde a un niño del sexo masculino ,al respecto se señala que esta enfermedad puede desarrollarse a cualquiera edad pero la incidencia es más al ta en niños y adultos jóvenes, siendo los varones más afectados que las mujeres.

La localización del microorganismo en la mucosa nasal coincide con el sitio más frecuentemente afectado reportado por la literatura médica. La eliminación del material purulento por la naríz no es frecuente, pero puede existir rinitis aguda, estenosis nasal parcial o completa, la perdida de sangre es aún más rara (8). En este paciente lo primero que se constató fue el sangramiento por la naríz La herida cicatrizó por primera intención , sin embargo presentó recidiva , ya que no cauterizó la primera vez.Por este motivo se recomienda la utilización de distermia y electrocoagulación y diferentes causticos como el antimonio pentavalente, nitrato de plata $(10,11$.).Se señala que el Dapsone aplicado por un año logra la curación de los casos( 12).

\section{REFERENCIAS}

1. Vander-Coer, J.M.; Marres, W.E.N.; Wong-Alcala, L.S. (1992). Rhinosporidiosis in Europe. J. Laryngol. Otol. 106: 440-443

2. Acha, P. \& Syfres, B. (1986). Zoonosis y enfermedades trasmisibles comunes al hombre y a los animales. Segunda edición. Publicación cientifica $\mathrm{N}^{\circ}$ 503, Washington. Editorial Organización Panamericana de la Salud. P

3. Ahlawalia, K.B. (1992). New interpretations in Rhinosporidiosis enigmatic disease of the last nine decades. J. Submicroc. Cytol. Pathol. 24: 109114

4. Mears, T.; Amerasinghe, C. (1992). Rhinosporidiosis. J. Laryngol. Otol. 106: 468

5. Kamal,M.M.; Luley,A.S.; Mundhada,S.G. \& Bobhate, S.K. (1995). Rhinosporidiosis. Diagnosis by scrape cytology. Acta Cytologica 39:931935

6. Gori, S.; Scasso, A. (1994). Cytologic and differential diagnosis of Rhinosporidiosis. Acta. Cytol. 38: 361-366
7. Batsakis, J.G.; El-Naggar, A.K. (1992). Rhinoscleroma and Rhinosporidiosis. Ann. Otol. Rhinol. Laryngol. 101: 879-882

8. Rathakar, C.; Madhavan, M.; Sankaran, V.; Veliath, A.J.; Majunder, N.K.; Rao, V.A. (1992). Rhinosporidiosis in Pondicherry. J. Trop. Med. Hyg. 95: 280-283

9. Gómez, R.; Pérez, A. (1971). Rhinosporidiosis ocular. Rev. Cub. Med. 10:541-546

10. Ballenger, J. (1984). Enfermedades de la nariz, garganta y oido. Tomo I, La Habana, Editorial Cientifico- Técnica. pp 310-311

11. Ravi, R.; Mallikarjuna, V.S.; Chaturvedi, H.K. (1992). Rhinosporidiosis micking penile malignancy. Urol. Int. 49:224-226

12. Job, A.; Venkateswaran, S.; Mathan, M.; Krishnawami, H.; Raman,R. (1993). Medical therapy of Rhinosporidiosis with Dapsone. J. Laryngol. Otol. 107: 809-812 